\title{
Efficient egalitarian equivalent allocations over a single good
}

\author{
Marco LiCalzi · Antonio Nicolò
}

Received: 20 June 2007 / Accepted: 20 March 2008 / Published online: 4 April 2008

(C) Springer-Verlag 2008

\begin{abstract}
This paper studies efficient and egalitarian allocations over a single heterogeneous and infinitely divisible good. We prove the existence of such allocations using only measure-theoretic arguments. Under the additional assumption of complete information, we identify a sufficient condition on agents' preferences that makes it possible to apply the Pazner-Schmeidler rule for uniquely selecting an efficient egalitarian equivalent allocation. Finally, we exhibit a simple procedure that implements the Pazner-Schmeidler selection in a subgame-perfect equilibrium.
\end{abstract}

Keywords Egalitarism $\cdot$ Equity $\cdot$ Fair division $\cdot$ Implementation $\cdot$ No-envy

JEL Classification $\quad \mathrm{D} 63 \cdot \mathrm{C} 70 \cdot \mathrm{D} 30$

\section{Introduction}

A fair division problem arises when two or more agents are called to divide a good over which they claim equal rights. The oldest known examples include Abraham and Lot arguing over land division (Genesis 13), and Prometheus and Zeus disputing a pile of meat (Hesiod's Theogony). A recent dramatic example is the carving of Bosnia

We thank Gabrielle Demange, William Thomson, two referees and seminar audiences at Boston, Palermo and Rochester University for their comments. Financial support from MIUR is acknowledged.

M. LiCalzi (凶)

SSE and Department of Applied Mathematics, University Ca'Foscari of Venice, 30123 Venice, Italy e-mail: licalzi@unive.it

\section{A. Nicolò}

Department of Economics, University of Padua, 37123 Padua, Italy

e-mail: antonio.nicolo@unipd.it 
and Herzegovina as an independent entity within the Dayton Accords that put an end to a 3-year civil war over the spoils of the former Republic of Yugoslavia.

There are several situations where the solution of a fair division problem cannot call on instruments like prices, monetary compensations, or auctions. This may be due to liquidity constraints; or to the psychological difficulty of bringing a dispute down to monetary evaluations; or to political constraints, as in the case of Bosnia and Herzegovina; or to the presence of judicially enforceable rights — such as under U.S. law- "to seek partition in kind, or physical division, of jointly owned land"; see Miceli and Sirmans (2000).

This paper studies the problem of fair division when the dispute must be resolved using division in kind. We are interested in devising a procedure that can help the parties to reach an outcome that is both fair and efficient. We assume that the disputed object is a single infinitely divisible good over which agents have heterogeneous preferences and that there are no consumption externalities. The canonical example is the division of a cake, when agents have different (additive) preferences over different slices; see Steinhaus (1948). A less obvious example is the case of a finite (or countable) number of homogeneous infinitely divisible goods, where the aggregate endowment is viewed as the single heterogeneous good at stake; see Chambers (2005, Sect. 5).

There are two main ordinal concepts in the fair division literature. The first is the envy-free principle which states that each party should (weakly) prefers its share to anyone else's. This was proposed by Gamow and Stern (1958, pp. 117-119), but became widely known after Foley (1967). Any efficient envy-free allocation is ex post stable because no one desires to exchange what he received with anyone else's share. However, this solution concept suffers from a multiplicity problem that makes it less satisfactory from an ex-ante, or procedural, point of view. There are in general many efficient envy-free allocations, and each of them provides different payoffs to the agents. Therefore, they are likely to disagree on how to select one among these allocations. The divide-and-choose mechanism under complete information, for instance, selects among all the efficient envy-free allocations the division that maximizes the payoff to the divider-so conflict is likely to shift over how the divider is chosen.

An alternative normative concept is the egalitarian equivalent criterion which states that each party should be indifferent between getting his share and some reference bundle, identical for all agents. This was introduced by Pazner and Schmeidler (1978) to overcome the problem that efficient no-envy allocation may not exist at all for economies with nonconvex preferences or with production. As different reference bundles lead to different shares, the multiplicity problem over efficient and egalitarian equivalent allocations resurfaces in the choice of the reference bundle. Pazner and Schmeidler (1978) suggests circumventing the difficulty by focusing only on those reference bundles that are proportional to the total endowment. (Assuming efficiency, this leads to a unique selection.) Sprumont and Zhou (1999) axiomatizes this "Pazner-Schmeidler" rule for large exchange economies with convex preferences where the endowment is a finite number of homogeneous infinitely divisible goods.

It is not immediately obvious how to extend the "Pazner-Schmeidler" rule when the endowment is a single heterogeneous good. Consider the division of a contested cake among a group of people who have equal claims on it. The agents may evaluate the value of a piece of the cake along different attributes: its crust, its filling, its weight, 
the number of strawberries on it, and so on. The challenge is how to make sure that all relevant attributes are proportionally represented in the reference bundle. Moreover, even if the parties themselves agree that a criterion should be represented whenever an agent cares about it, is there a way to elicit this strategic information from each party?

We answer these questions under the assumption that each agent can partition the disputed cake into a finite (or countable) number of parcels that he (but not necessarily the other parties) views as homogeneous. The intuition is the following. Each agent divides the cake in as many parcels as he likes. Equally sized morsels from the same parcel carry the same utility to the agent, so that each parcel is a homogeneous good for the agent. Note that equally sized morsels from two different parcels may carry different utility to him; and, similarly, equally sized morsels from an agent's parcel may give different utilities to another agent. Consider now the common refinement of all the agents' partitions. Each parcel in this new and finer partition is a homogeneous good for each party. This brings us back to the standard setting for the Pazner-Schmeidler rule. Hence, we choose the reference bundle among those that are proportional to this common refinement. Under efficiency, the selection of the reference bundle to define the egalitarian equivalent allocation is again unique.

Clearly, in the search for a procedure to implement the efficient egalitarian equivalent allocation with respect to this special reference bundle, we also need to overcome the difficulty of devising a game in which each agent reveals his own partition of the cake. Lying over one's partition may lead to a different reference bundle and hence to a better share for the liar. We provide a simple procedure which implements the desired outcome as a subgame-perfect equilibrium, under the assumption that agents have complete information about their preferences. The procedure is simple in the sense of Thomson (2005). It generalizes a mechanism suggested in Crawford (1979) and ameliorated in Demange (1984). Their mechanism derives an efficient egalitarian equivalent allocation for a finite collection of homogenous goods. Our procedure must also simultaneously discover the right way to partition the heterogeneous good.

The paper is organized as follows. Section 2 describes our model, which is a standard version of the classical setup for cake division problems. Section 3 proves the existence of efficient egalitarian equivalent allocations for a single heterogeneous good using only measure-theoretic assumptions; the only other existence result we are aware of is more general in scope but requires additional topological assumptions; see Berliant et al. (1992). Section 4 describes the assumptions that define the economic environment over which our procedure can be applied. Section 5 states the implementation result. Section 6 contains a brief closing remark. Long proofs are relegated to the Appendix.

\section{The model}

Our model is an abstraction of the classical problem where a cake (or a piece of land) must be allocated among several agents. There is a measurable space $(\Omega, \mathcal{F})$, where $\Omega$ is the object to be divided among the $n$ agents and $\mathcal{F}$ is a $\sigma$-algebra over $\Omega$. We say that an element of $\mathcal{F}$ is a parcel and that an $\mathcal{F}$-measurable subset of a parcel is a morsel, which is a nicer term than "subparcel". Any subset of $\Omega$ mentioned in the following is an element of $\mathcal{F}$, and hence a parcel. 
For $n \geq 2$, let $N=\{1,2, \ldots, n\}$ be the (finite) set of agents. Agents have preferences over parcels of $\Omega$. Each agent $i$ is endowed with a utility function $u_{i}$ : $\mathcal{F} \rightarrow \mathbb{R}^{+}$that is a nonatomic probability measure on $\mathcal{F}$. (Since preferences are invariant up to a positive rescaling of the utility function, $u_{i}(\Omega)=1$ is only a normalization.) A measure $u_{i}$ is nonatomic if, for each parcel $A$ and each $x$ in $(0, u(A))$, there exists another parcel $B \subseteq A$ such that $u_{i}(B)=x$. Hence, the range of each $u_{i}$ is the (convex) interval $[0,1]$.

A utility function $u$ over parcels is absolutely continuous with respect to another measure $\mu$ over $\mathcal{F}$ if $\mu(A)=0$ implies $u(A)=0$ for any parcel $A$. Clearly, any utility function $u_{i}$ is absolutely continuous with respect to the measure $\mu=\sum_{i=1}^{n} u_{i}$. We make the assumption that the utility functions are mutually absolutely continuous; that is, if $u_{i}(A)=0$ for some parcel $A$, then $u_{j}(A)=0$ for any agent $j$. Since agents agree on the null parcels, we say that a parcel has zero (or positive) measure without specifying a measure.

An allocation $X=\left(x_{1}, \ldots, x_{n}\right)$ is a partition of $\Omega$ in $n$ parcels, where $x_{i}$ is the parcel assigned to agent $i$ in $N$. An allocation $X$ is efficient (or weakly efficient, respectively) if there exists no other allocation $Y=\left(y_{1}, \ldots, y_{n}\right)$ such that $u_{i}\left(y_{i}\right) \geq u_{i}\left(x_{i}\right)$ for all $i$, with the strict inequality holding for some $i$ (or $u_{i}\left(y_{i}\right)>u_{i}\left(x_{i}\right)$ for all $i$ ). Any efficient allocation is also weakly efficient. The converse is true under our assumption that agents have preferences that are mutually absolutely continuous; see Akin (1995, Lemma 9).

There are several criteria to evaluate the fairness of an allocation. For instance, an allocation $X$ is proportionally fair if $u_{i}\left(x_{i}\right) \geq(1 / n)$ for all $i$; and it is equitable if $u_{i}\left(x_{i}\right)=u_{j}\left(x_{j}\right)$ for all $i$ and $j$. These two notions of fairness hinge on the demanding assumption that interpersonal preferences are comparable. The main fairness criteria based on ordinal preferences are two. An allocation $X$ is envy-free if $u_{i}\left(x_{i}\right) \geq u_{i}\left(x_{j}\right)$ for all $i$ and $j$, and it is egalitarian equivalent (for short, $\mathrm{EE}$ ) if there exists a reference parcel $A$ such that $u_{i}\left(x_{i}\right)=u_{i}(A)$ for all $i$. Any envy-free allocation is proportionally fair, but the converse is true only if $n=2$.

Under our setup, the following existence results are known. Dubins and Spanier (1961) proves the existence of efficient and proportionally fair allocations for preferences which may not be mutually absolutely continuous. It notes that adding this latter assumption ensures that all efficient allocations are equitable. Maccheroni and Marinacci (2003) gives sufficient conditions to extend the existence result for proportionally fair allocations when the utility functions are concave capacities. Weller (1985) proves the existence of weakly efficient and envy-free allocations; efficiency follows immediately under mutual absolute continuity.

More existence results are known under related setups, which additionally assume that $\Omega$ is a subset of $\mathbb{R}^{k}$. For instance, Stromquist (1980) proves the existence of envyfree allocations for a planar cake using a larger class of preferences, but restricting the set of admissible partitions. Berliant et al. (1992) has several results. It gives a stronger version of Weller (1985) result assuming that the utility functions are absolutely continuous with respect to the Lebesgue measure. And it proves the existence of efficient and egalitarian equivalent allocations for a general class of preferences that must however be continuous in a complicated topology described in Berliant and Dunz (2004). 


\section{Existence of efficient EE partitions}

This section proves the existence of efficient and egalitarian equivalent allocations in our setup. Existence follows already from Theorem 5 in Berliant et al. (1992) but our proof requires no topological assumptions and extends to more general domains.

We need a few definitions. Let $u=\left(u_{1}, \ldots, u_{n}\right)$ be the vector of the $n$ agents' utility functions on the measurable space $(\Omega, \mathcal{F})$. The set $R(u)=\{u(A): A \in \mathcal{F}\}$ in $\mathbb{R}^{n}$ is the range of $u$. The range of $u$ spans the vector of utilities that the agents can achieve if they are all given the same parcel. By assumption, each $u_{i}$ is a nonatomic probability measure. Then, by Lyapunov's convexity theorem, $R(u)$ is a compact and convex subset of $\mathbb{R}^{n}$.

Let $\Pi$ be the set of all $n$-partitions of $\Omega$. The set $R P(u)=\left\{\left(u_{1}\left(x_{1}\right), \ldots, u_{n}\left(x_{n}\right)\right)\right.$ : $X \in \Pi\}$ in $\mathbb{R}^{n}$ is the partition range of $u$. The partition range is sometimes called the Individual Pieces Set; see Barbanel (2005). The partition range of $u$ spans the vector of utilities that the agents can achieve by dividing up the cake according to some allocation. Dvoretzky et al. (1951) derives from Lyapunov's convexity theorem a more general result, which implies that the partition range is also a compact and convex subset of $\mathbb{R}^{n}$.

We also call on the following three lemmata, which assume that $u$ is a (finite) vector of nonatomic probability measures. The first two results correspond to Corollary 1.1 and Lemma 5.3 in Dubins and Spanier (1961), respectively. They do not require preferences that are mutually absolutely continuous.

Lemma 1 Given an integer $k$ and positive weights $\alpha_{1}, \ldots, \alpha_{k}$ with $\sum_{j} \alpha_{j}=1$, there exists a $k$-partition $X=\left(x_{1}, \ldots, x_{k}\right)$ such that $u_{i}\left(x_{j}\right)=\alpha_{j}$ for all $i=1, \ldots, n$ and $j=1, \ldots, k$.

For $k=n$ and $\alpha_{j}=1 / n$ for all $j$, this implies that the partition range of $u$ always contains the point $(1 / n, \ldots, 1 / n)$. The next lemma, instead, concerns the range of $u$ and implies that it always contains the whole diagonal.

Lemma 2 For any $t$ in $[0,1]$ there exists a parcel $A_{t}$ such that $u_{i}\left(A_{t}\right)=t$ for each $i$.

Our last lemma characterize efficient allocations when preference are mutually absolutely continuous; see Barbanel and Zwicker (1997, Theorem 1). Section 7C in Barbanel (2005) discusses the case where mutual absolute continuity does not hold.

Lemma 3 If preferences are mutually absolutely continuous, an allocation is efficient if and only if it maximizes a convex combination of the utility functions.

The following is the main result in this section.

Theorem 1 There exists at least one allocation which is efficient and egalitarian equivalent.

Proof Consider the set $R P(u)$. If the vector $\mathbf{1}=(1, \ldots, 1)$ belongs to $R P(u)$, there exists an allocation such that every agent has utility $1=u_{i}(\Omega)$. Clearly, this allocation is efficient and egalitarian equivalent with respect to the reference parcel $\Omega$. 
So, assume that the vector $\mathbf{1}$ is not in $R P(u)$. For any $t$ in $[0,1]$, let $\mathbf{t}=(t, \ldots, t)$. By Lemma 1, $R P(u)$ contains $\mathbf{1} / \mathbf{n}$ and so its intersection with the diagonal is not empty. Therefore, the continuous function $f(\mathbf{t})=t$ attains a maximum $t^{*}<1$ on this intersection. The vector $\mathbf{t}^{*}$ is in $R P(u)$, so there exists some allocation $X^{*}$ such that $u_{i}\left(x_{i}^{*}\right)=t^{*}$ for each $i$. By Lemma 2, there exists some parcel $A$ such that $u_{i}(A)=t^{*}$ and thus $X^{*}$ is an egalitarian equivalent allocation with respect to the reference parcel $A$.

Moreover, since $\mathbf{t}^{*}$ is also a boundary point of the convex set $R P(u)$, there is a supporting hyperplane for $R P(u)$ going through $\mathbf{t}^{*}$. Thus, for some convex combination of weights $\left(\alpha_{1}, \ldots, \alpha_{n}\right), \sum_{i} u_{i}\left(x_{i}^{*}\right)=\sum_{i} \alpha_{i} t^{*} \geq \sum_{i} \alpha_{i} u_{i}$ for any $u$ in $R P(u)$ and hence by Lemma $3 X^{*}$ is also efficient.

\section{An economic environment for EE allocations}

The existence result in Theorem 1 is not constructive. In other words, it does not tell us how to find $X^{*}$. It does not even try and ask agents if they know what $X^{*}$ should be. This leads naturally to the question of designing a procedure that generates an efficient and egalitarian equivalent allocation.

Crawford (1979) provides a solution to this problem when there is a finite number of perfectly divisible homogeneous goods, under the assumption that there is complete information about agents' (continuous and strongly monotonic) preferences. Given a numeraire bundle $x$ that is desirable for all agents, each agent bids a price for the right to propose the allocation. If everybody accepts the winner's proposal, this is carried out. If an agent refuses the proposal, the final allocation is derived from the equal share rule in which everybody gets $(1 / n)$ of the original endowment as follows: the divider gives up a fraction $p$ (equal to his bid) of $x$ that is equally shared among the other agents.

As it turns out, there is a unique price $p^{*}$ which makes every agent indifferent between the roles of divider and chooser. The procedure generates a final allocation that is efficient and egalitarian equivalent with respect to the reference bundle formed by the union of a fraction $(1 / n)$ of the original endowment and a fraction $p^{*}$ of the numeraire bundle. Demange (1984) improves on this scheme by proposing a version that avoids the infeasible off-equilibrium allocations present in Crawford (1979) procedure.

When viewed as an implementation result for their economic environment, these results exhibit a limitation. Although the choice of the numeraire bundle affects the final allocation, the procedure assumes that the numeraire bundle is given exogenously, circumventing the problem of how agents come to agree on it. Crawford (1979) points out that either plain money or a bundle proportional to the total endowment are likely to be focal choices for the numeraire bundle. This latter choice defines the PaznerSchmeidler rule for the selection of the numeraire bundle, as axiomatized in Sprumont and Zhou (1999).

In our setup with just one heterogenous good, there is no money and it is not clear how to define a proportional bundle. Our contribution in this section is to identify an economic environment where the definition of a proportional bundle should be 
uncontroversial, making the use of the Pazner-Schmeidler rule intuitively natural. We then extend the Crawford-Demange procedure accordingly and provide a general method to achieve efficient and egalitarian equivalent allocations for a single heterogeneous good. ${ }^{1}$

We enrich the setup in Sect. 2 with three assumptions, that define the economic environment investigated in this section.

(A1) There is a measure $\mu$ on the measurable space $(\Omega, \mathcal{F})$.

(A2) For each $i$, the utility $u_{i}$ is absolutely continuous with respect to $\mu$.

(A3) For each $i$, there exists a finite partition $P^{i}=\left(p_{1}^{i}, \ldots, p_{m_{i}}^{i}\right)$ such that, if two parcels $A, B$ satisfy $A \cup B \subseteq p_{j}^{i}$ then

$$
\mu(A)=\mu(B) \Rightarrow u_{i}(A)=u_{i}(B) .
$$

Assumption (A1) requires that there is a common "objective" measure. If $\Omega$ is a subset of $\mathbb{R}^{k}$, this might be the Lebesgue measure. Assumption (A2) requires that an agent attaches no utility to sets that have size zero, where the size of a parcel is simply its $\mu$-measure. Finally, Assumption (A3) states that there is a partition $P^{i}$ which divides $\Omega$ into $m_{i}$ parcels, each of which can be considered a homogeneous good for agent $i$. Assumption (A3) can be considerably relaxed by allowing $P^{i}$ to be constituted by a countably infinite number of parcels. After few obvious modifications, all of our results would still hold. So it is only in the interest of simplicity that we assume that $P^{i}$ is finite.

An example may help to assess the import of these three assumptions. Suppose that the object to be allocated is a chocolate chip cookie, which we may think of as a subset of $\mathbb{R}^{3}$. Presumably, agents care only about the cookie dough or the chocolate chips, although possibly in different guises. Then (A1) is satisfied by taking $\mu$ to be the Lebesgue measure on $\mathbb{R}^{3}$ and (A2) holds for instance if agents attach no utility to morsels with empty interior. Finally, (A3) is satisfied by assuming that each agent partitions the cookie into two sets: the dough and the chips. From a technical viewpoint, we note that, by the Radon-Nikodym Theorem, (A1)-(A3) jointly state that we can write the utility function of each agent $i$ as $u_{i}(A)=\int_{A} f_{i} \mathrm{~d} \mu$, where $f_{i}$ is a finitely (or countably)-valued density.

While (A1) and (A3) may look restrictive when presented in an abstract setting, we have not been able to find applications in which it is not natural to assume that there is an objective way to measure $\Omega$ and that an agent cares differently about the parcels he gets in more than a finite (or countable) number of ways. Even the standard example of an exchange economy with a finite (or countable) number of homogeneous goods implicitly assumes both (A1) and (A3); see Chambers (2005, Sect. 5). Sprumont (2004) axiomatizes a closely related notion of cardinal commodities, and Section 6 comments more on the import of (A3). Assumption (A2) is a more technical condition which we need only to ensure the efficiency of a specific family of allocations defined below; see Footnote 2.

\footnotetext{
1 In this respect, Demange (1984) suggests a lottery-based extension of the procedure, but its demanding assumptions impose that agents be expected utility maximizers and that the whole good be randomly assigned to a single agent off the equilibrium path.
} 
Any finite refinement of $P^{i}$ defines another partition that satisfies (A3). Ordering such finite partitions by inclusion defines a lattice on the set of partitions of $\Omega$. We denote by $P_{1} \vee P_{2}$ and $P_{1} \wedge P_{2}$ the common coarsening and the common refinement of two partitions $P_{1}$ and $P_{2}$, respectively.

For definiteness, we associate each agent $i$ with an individual partition $P_{i}^{c}$ that is the common coarsening of all finite partitions that satisfy (A3). Clearly, the individual partition $P_{i}^{c}$ is the only partition among those satisfying (A3) for which any two parcels $A \subseteq p_{j}^{i}$ and $B \subseteq p_{l}^{i}(j \neq l)$ such that $\mu(A)=\mu(B)$ are associated with distinct utilities $u_{i}(A) \neq u_{i}(B)$.

Consider now the common refinement $P^{c}=\bigwedge_{i \in N} P_{i}^{c}$ of the individual partitions for each agent, which we call the natural partition for $\Omega$. This finer partition extends (A3) to all agents, in the sense that if two parcels $A, B$ satisfy $A \cup B \subseteq p_{j}$ in $P^{c}$ then

$$
\mu(A)=\mu(B) \Rightarrow u_{i}(A)=u_{i}(B) \text { for all } i \text { in } N .
$$

Therefore, the natural partition $P^{c}$ of $\Omega$ divides the original heterogeneous good in a finite set of parcels which each player views as homogeneous. Clearly, any common refinement of arbitrary partitions that satisfy (A3) for all $i$ leads to a common partition finer than $P^{c}$ for which (1) still holds. The natural partition $P^{c}$ is simply the smallest one among those that satisfy (1): focusing on it entails no loss of generality.

Let $P=\left(p_{1}, \ldots, p_{m}\right)$ be the natural partition (or a refinement of it). Then each agent is indifferent among morsels of equal size from a single parcel $p_{j}$ and we can construct a bundle by adjoining proportional morsels from each parcel of the natural partition. Formally speaking, a proportional bundle of size $\lambda$ obtains if we choose $\lambda$ in $[0,1]$ and then pick a morsel $e_{j}$ from each parcel $p_{j}$ such that $\mu\left(e_{j}\right)=\lambda \mu\left(p_{j}\right)$ for all $j=1, \ldots, m$. The proportional bundle is $A=\bigcup_{j=1}^{m} e_{j}$ and provides each agent with utility $\lambda \mu(\Omega)=\lambda$. Note that we do not specify how a morsel $e_{j}$ is chosen from the parcel $p_{j}$ because, as far as its size $\mu\left(e_{j}\right)=\lambda \mu\left(p_{j}\right)$, each agent is indifferent over the actual morsel chosen. This makes it irrelevant to know the identity of the divider who actually gets to choose the morsels from a parcel.

If $\sum_{i} \lambda_{i}=1$, we can collect $n$ proportional bundles to derive a proportional allocation that gives each agent $i$ a bundle of size $\lambda_{i} \geq 0$, from which he derives utility $\lambda_{i}$. Suppose again that $P=\left(p_{1}, \ldots, p_{m}\right)$ is the natural partition (or a refinement). Construct $n$ proportional bundles of size $\lambda_{i}$ for each agent $i=1, \ldots, n$ by choosing (or instructing a divider to choose) morsels of size $\lambda_{i} \mu\left(p_{j}\right)$ from each parcel $p_{j}$ in $P$. Since $\sum_{i} \lambda_{i}=1$, the divider can pick mutually exclusive morsels from each parcel that jointly exhaust $\Omega$ (up to a $\mu$-null set $^{2}$ ). This results into a proportional allocation that splits $\Omega$ into $n$ proportional bundles such that the bundle of size $\lambda_{i}$ gives utility $\lambda_{i}$ to agent $i$. In particular, when $\lambda_{i}=1 / n$ for all $i$, this allocation is proportional and envy-free but in general inefficient.

The next section shows how to implement egalitarian equivalent allocations with respect to a reference parcel that is a proportional bundle of size $\lambda^{*} \geq 1 / n$. This is the

\footnotetext{
2 By (A2), a residual set of size zero carries no utility for any agent. From now on, we assume without loss of generality that no residual set of size zero is left over.
} 
case of interest when all agents have equal claims on the good to be divided. The title of the next section is a pun on similar titles in the literature.

\section{How to cut a cookie fairly}

We exhibit a procedure that implements an efficient and egalitarian equivalent allocation when the object to be divided is a heterogeneous good that is infinitely divisible, in the setup of Sect. 2. We assume complete information about preferences, as well as (A1)-(A3). The solution concept is subgame perfection, as defined in Sect. 1c of Demange (1984). Under a bland tie-breaking assumption, we show that there are unique equilibrium payoffs, with a final allocation that is efficient, proportionally fair and egalitarian equivalent.

The procedure is inspired by the mechanism described in Demange (1984) to improve the version proposed in Crawford (1979). We have found a few different game forms that do the job. The one presented here is especially expedient because on the equilibrium path the only messages announced are $n$ bids (one for each agent), the final allocation as chosen by an agent (called divider), and $n-1$ "yes" from the remaining agents (called choosers) who accept the divider's proposal; off the equilibrium path, the worst case has a chooser saying "no", after which each agent announces a partition and the final allocation is a suitable proportional allocation where each player picks his own parcel. The procedure develops in two stages.

Stage 1. Each agent $i$ in $N$ simultaneously announces a bid $b_{i}$ between $1 / n$ and 1 . Agents are ordered by decreasing bids using if necessary an exogenous (nonrandom) tie-breaking rule, so that $b_{1} \geq b_{2} \geq \cdots \geq b_{n}$. Agent 1 is called the divider, while any other agent is a chooser.

Stage 2. The last stage consists of $n$ consecutive moves, one for each agent.

Move 0. The divider proposes an allocation $X$.

Move 1. Chooser $n$ accepts or refuses. If he accepts, move 2 is played. If he refuses, each agent $i$ announces ${ }^{3}$ a partition $P^{i}$ and the players select ${ }^{4}$ a proportional allocation $X^{n}$ based on the common partition $\bigwedge_{i=1}^{n} P^{i}$ and the vector of weights $\lambda^{n}$ defined below; ${ }^{5}$ then the game stops and $X^{n}$ is the final allocation.

Move $i+1$. Chooser $n-i$ accepts or refuses. If he refuses, each agent $i$ announces a partition $P^{i}$ and the players select a $\lambda^{n-i}$-proportional allocation $X^{n-i}$ over the

\footnotetext{
3 The timing of these announcements is irrelevant for the equilibrium outcome.

4 We assume that, using the pecking order defined below, each chooser sequentially removes his own proportional bundle from $\Omega$; the divider gets what remains.

5 For short, we call this a $\lambda^{n}$-proportional allocation in the following.
} 
partition $\bigwedge_{i=1}^{n} P^{i}$; then the game stops and $X^{n-i}$ is the final allocation. If he accepts, move $i+2$ is played.

Move $n-1$. Chooser 2 accepts or refuses. If he refuses, each agent $i$ announces a partition $P^{i}$ and the players select a $\lambda^{2}$-proportional allocation $X^{2}$ over the partition $\bigwedge_{i=1}^{n} P^{i}$; then the game stops and $X^{2}$ is the final allocation. If he accepts, the game stops and the final allocation $X$ is the divider's proposal.

If agent $i$ is the first to refuse the divider's proposal, this affects the choice of the vector $\lambda^{i}$ of convex weights used by the same agent $i$ to pick a proportional allocation. These vectors of convex weights are defined hereafter.

Proportionality weights. To construct the vectors $\lambda^{i}$ (for $i=2, \ldots, n$ ) of proportionality coefficients, we define a pecking order for agents. Suppose that players sit around a circle arranged in standard (clockwise) increasing order. When $i$ refuses, the circle is walked counterclockwise starting from $i$ and the pecking order is $i<i-1<$ $\cdots<2<n<n-1<\cdots<i+1$. Let $\sigma^{i}(k)$ denote the $k$ th agent in this pecking order. We are ready to define the vector $\lambda^{i}=\left(\lambda_{1}^{i}, \ldots, \lambda_{n}^{i}\right)$ used if $i$ refuses the proposed allocation. The refusing agent $i=\sigma^{i}(1)$ is assigned the proportionality coefficient $\lambda_{i}^{i}=b_{i}$. Following the pecking order, a later chooser $\sigma^{i}(k)$ is assigned the coefficient

$$
\lambda_{\sigma^{i}(k)}^{i}= \begin{cases}b_{\sigma^{i}(k)}, & \text { if } \sum_{s=1}^{k} b_{\sigma^{i}(s)} \leq 1 \\ \max \left(0,1-\sum_{s=1}^{k-1} b_{\sigma^{i}(s)}\right), & \text { otherwise. }\end{cases}
$$

In other words, later choosers are assigned a proportionality coefficient equal to their bid when this is still a feasible convex weight, or otherwise its truncation to 1 . Whenever there is a refusal, the divider is assigned the (same) coefficient $\lambda_{1}^{i}=$ $\max \left(0,1-\sum_{s=2}^{n} b_{s}\right)$, regardless of the refuser $i$ 's identity; for short, we denote this by $\beta_{1}$. For each $i$, the positive weights in $\lambda^{i}$ add up to 1 , so that (up to a set of size zero, irrelevant by (A2)) the $\lambda^{i}$-proportional allocation can be made to exhaust the whole good.

This concludes the description of the procedure. We prove two results in this section. The first one is that equilibrium allocations must be proportionally fair, in the sense that each agent receives a parcel which carries utility of at least $1 / n$ to him. The second and main result is that this procedure has unique equilibrium payoffs and yields an allocation $X$ that is efficient and egalitarian equivalent.

We need a few definitions. Let $\mathcal{A}$ be the set of all possible allocations. Given a partition $P$ and a vector $\lambda$ of convex weights, let $\Pi(\lambda \mid P)$ denote the set of $\lambda$-proportional allocations over $P$. Clearly, this set contains (uncountably) many possible allocations. When $P$ is the individual partition $P_{i}^{c}$ or any of its refinements (such as the natural partition $\left.P^{c}\right)$, the agent is indifferent over any allocation in $\Pi(\lambda \mid P)$ and thus his utility from a $\lambda$-proportional allocation over $P$ is well-defined.

Theorem 2 Any equilibrium allocation for the procedure described above is proportionally fair. 
Proof We prove the stronger statement that each agent has a strategy that guarantees him the utility level $\bar{u}=(1 / n)$ associated with the proportional allocation $\Pi\left(\frac{1}{n} \mathbf{1} \mid P^{c}\right)$. This implies that in equilibrium an agent must achieve at least utility $\bar{u}=(1 / n)$.

The strategy is the following. The agent bids $(1 / n)$ in the first stage. In the second stage, if he is the divider, he chooses the $\frac{1}{n} \mathbf{1}$-proportional allocation over $P_{i}^{c}$; if he is a chooser, he refuses any proposed allocation; whenever asked for, he announces the partition $P_{i}^{c}$.

If the agent ends up being the divider and his proposal is accepted, the final allocation is in $\Pi\left(\frac{1}{n} \mathbf{1} \mid P_{i}^{c}\right)$ and his utility is $\bar{u}=(1 / n)$. If his proposal is refused by an agent $j$, announcing $P^{1}=P_{i}^{c}$ implies that $\bigwedge_{i=1}^{n} P^{i}$ is not coarser than $P_{i}^{c}$ and thus the final allocation is in $\Pi\left(\lambda^{j} \mid P_{i}^{c}\right)$. But $\lambda^{j}=\frac{1}{n} \mathbf{1}$, because if the divider's bid is $(1 / n)$ all other bids are equal to $(1 / n)$. Hence, the final allocation is again in $\Pi\left(\frac{1}{n} \mathbf{1} \mid P_{i}^{c}\right)$ and the divider gets utility $\bar{u}=(1 / n)$.

On the other hand, if the agent is chooser $i$, then every agent $n, n-1, \ldots, i+1$ before him in the pecking order has bid $b_{j}=(1 / n)$. So, if a chooser $j=i, \ldots, n$ refuses, the vector $\lambda^{j}$ has $\lambda_{k}^{j}=(1 / n)$ for $k=i, \ldots, n$. Hence, if a refusal occurs up to (and including) $i$ 's move, the final allocation is in $\Pi\left(\lambda^{j} \mid P_{i}^{c}\right)$ which by a reasoning similar to the above yields utility $\bar{u}=(1 / n)$ to agent $i$.

It is worth noting that the strategy discussed in the above proof requires the agent to use and announce only information about his own preferences; therefore, this strategy remains viable even when he has no information about other agents' preferences. Hence, the ability of each agent to secure a proportionally fair allocation is a robust feature that does not depend on our assumption of complete information.

The following is the main result in this section. Its proof, including a few lemmata, can be found in the Appendix. We assume that a chooser who is indifferent always prefers the move that keeps his play simpler and, subordinately, the move that ends the game sooner; for instance, a tie between accepting or refusing a proposal is broken by accepting because this spares him the need to announce a partition.

The statement uses the following piece of notation. Let $\Pi\left(\lambda \mid P^{c}\right)$ be the set of $\lambda$-proportional allocations over the natural partition $P^{c}$ (or a refinement) and let $\pi_{i}\left(\lambda \mid P^{c}\right)$ denote the set of parcels that these allocations may assign to agent $i$. Recall that the equilibrium concept used is subgame perfection; for short, we simply speak of "equilibrium".

Theorem 3 The procedure described above has unique equilibrium payoffs, with final allocations that are efficient and egalitarian equivalent. In every equilibrium allocation, each agent $i$ is indifferent between the parcel he receives and getting $\pi_{i}\left(\lambda^{*} \mathbf{1} \mid P^{c}\right)$, where $\lambda^{*}=\max \left\{\lambda\right.$ : there exists $Y \in \mathcal{A}$ with $u_{i}\left(y_{i}\right) \geq u_{i}\left[\pi_{i}\left(\lambda \mathbf{1} \mid P^{c}\right)\right]$ for each $\left.i\right\}$.

The intuition for this result is the following. Our procedure asks each player to sequentially approve the allocation decided by the divider. When an allocation is not approved, each player $i$ receives a proportional share of (a common refinement for) the partition he declares. The utility of this proportional share depends on the partition used. However, by announcing his own individual partition, the player can make sure that he receives the same utility from his proportional share regardless of the common 
refinement that emerges from players' announcements. This sets a lower bound on the utility that a player must receive from the procedure. In the first stage, when players bid for the right to be the divider, they know that they will have to honor others' (credible) claims to these lower bounds out of their own share. This forces a unique equilibrium bid corresponding to an efficient and egalitarian equivalent allocation.

A simple example may help to illustrate this. Suppose that there are three agents $(n=3)$ and that the single good $\Omega$ to be allocated is the segment $[0,1]$, endowed with the usual Borel $\sigma$-algebra and Lebesgue measure $\mu$. Each agent $i=1,2,3$ likes the $i$-th third of the segment $[0,1]$ uniformly better than the rest. Formally, the utility of agent $i$ over a parcel $A$ is $u_{i}(A)=\int_{A} f_{i}(x) \mathrm{d} x$ where $f_{i}(x)=3 / 2$ for $x$ in the interval $[(1 / 3)(i-1),(1 / 3) i]$ and 3/4 otherwise. Clearly, (A1)-(A3) from Sect. 4 are satisfied.

There is a continuum of efficient allocations, but among these the equilibrium allocation using our procedure (up to a set of measure zero) assigns to each agent $i$ the parcel $[(1 / 3)(i-1),(1 / 3) i]$ he likes best. This allocation is proportionally fair because each agent derives a utility $1 / 2$ that is greater than $1 / 3$. And it is egalitarian equivalent with respect to the reference parcel $[0,1 / 6] \cup[2 / 6,3 / 6] \cup[4 / 6,5 / 6]$ that gives each agent a utility of $1 / 2$.

The game induced by our procedure has many payoff-equivalent equilibria. For the sake of the example, we describe here the gist of just one particularly simple equilibrium play. In stage 1 , each agent $i$ simultaneously announces $b_{i}=\frac{1}{2}$ so Agent 1 turns out to be the divider and he proposes an allocation $X$ where (up to a set of measure zero) each agent $i$ gets the $i$-th third of $[0,1]$. In stage 2 , Agent 3 and successively Agent 2 accept the allocation $X$, and this gets implemented. If someone refuses $X$, then 1 announces $P^{c}$. The equilibrium payoff is $u_{i}^{*}=1 / 2$ for each agent $i$.

The full argument requires a proof, but we can gather some intuition about the structure of the equilibrium from a few special cases. Consider stage 2: Agent 2 has no incentive to refuse $X$ because doing so would get her a proportional bundle of size $b_{2}=1 / 2$ from $P^{c}$ and hence a utility $1 / 2 \leq u_{2}^{*}$. Consider stage 1 : Agent 2 has no incentive to bid $b_{2}<1 / 2$, because 1 would steal a morsel of measure $1 / 2-b_{2}$ from her parcel [1/3,2/3] and reduce her utility. Moreover, Agent 2 has no incentive to bid higher to become divider herself: 1 and 3 accept her proposal only if they get a utility of at least $1 / 2$, which implies that she cannot get a utility higher than $1 / 2 \leq u_{2}^{*}$; and if her proposal is rejected, she gets a proportional bundle of size never greater than $1 / 2$ from $P^{c}$, which again cannot give her a utility higher than $u_{2}^{*}$.

\section{Closing remarks}

It may be argued that our assumption (A3) is too restrictive, because it transforms the original problem of dividing a heterogeneous good in a standard problem of dividing a finite (or countable) number of homogeneous goods. We have two comments in this respect.

First, our aim is to show that the problem of dividing a single heterogeneous good can be assimilated to the problem of dividing a bundle of homogeneous goods under assumptions that are not much demanding in practical applications. When players can 
use the partitions of $\Omega$ as a strategic variable, the complexity of their strategy space is humongous. However, if preferences satisfies (A3), the equilibrium is relatively easy to characterize.

Second, the main contribution is to suggest a way to endogenize the choice of the reference bundle that can be applied for the division of a bundle of goods when agents disagree about which are the characteristics that define a homogeneous good. Suppose that a donor bequest his painting collection to two museums, leaving to them the task of dividing the collection. In order to do so, the museums would like to construct a reference bundle that is a partition of the collection in homogeneous sets of works. A priori, there seems to be no "natural" way to construct this reference bundle. Should paintings be deemed homogeneous with respect to the century when they were painted, the nationality of the painters, or their artistic themes?

It seems only natural to let the museums decide the reference bundle according to their preferences. Assuming equal rights, this paper describes a mechanism that chooses this bundle endogenously. Each agent proposes a partition of the total endowment and the reference bundle is obtained as the common refinement of individual proposals. In equilibrium, this selects the natural partition (or a refinement) as the reference bundle.

\section{Appendix}

We recall and extend a piece of our notation to make the proofs in this Appendix easier to read. Let $\Pi(\lambda \mid P)$ denote the set of $\lambda$-proportional allocations over $P$ and let $\pi_{i}(\lambda \mid P)$ be the set of parcels that these allocations may assign to agent $i$. Since $\pi_{i}(\lambda \mid P)$ depends only on the $i$ th component of $\lambda$, we abuse notation and write simply $\pi_{i}\left(\lambda_{i} \mid P\right)$ when we need to highlight $i$ 's proportionality coefficient. If $P=P_{i}^{c}$ (or a refinement, ${ }^{6}$ and in particular the natural partition $\left.P^{c}\right)$, the agent is indifferent over any element in this set and we denote his utility by $v_{i}\left(\lambda_{i} \mid P_{i}^{c}\right)=u_{i}\left[\pi_{i}\left(\lambda_{i} \mid P_{i}^{c}\right)\right]$. By (A2) and the nonatomicity of $u_{i}, v_{i}\left(\lambda_{i} \mid P_{i}^{c}\right)$ is a strictly increasing continuous function of $\lambda_{i}$.

We assume that a chooser who is indifferent always prefers the move that keeps his play simpler and, subordinately, the move that ends the game sooner. Hence, when indifferent, he chooses acceptance over refusing the divider's proposal. When he is indifferent between refusing now and having some later chooser refuse, he prefers to refuse now.

The strategy of proof is the following. First, Lemmata 4-9 characterize the subgame perfect equilibrium where each agent announces $P^{c}$ in any continuation game after a refusal. Second, Lemma 10 shows that the payoffs generated by this equilibrium are efficient. Finally, Lemma 11 proves that there may not be other equilibrium payoffs.

In general, there are many equilibrium strategies that may be used in a continuation game after a refusal. The requirement that everybody announces $P^{c}$ in any continuation game is very strong, but we show later that any other equilibrium must achieve the same payoffs. The technical advantage of this assumption is apparent: when another

\footnotetext{
6 For simplicity, we leave it understood in the following that $P_{i}^{c}$ may be replaced by any of its refinements.
} 
player declares the partition $P^{c}$, the common partition to be used in the second stage is a refinement of $P_{i}^{c}$; hence, any partition announced by agent $i$ himself is payoff equivalent and thus is part of an equilibrium.

Lemma 4 Assume that the common partition in any continuation game after a refusal is $P^{c}$. Then a proposed allocation $X$ is accepted if and only if $u_{i}\left(x_{i}\right) \geq v_{i}\left(b_{i} \mid P^{c}\right)$ for each $i \geq 2$.

Proof The proof is by backwards induction. Suppose that the game has reached agent 2 . Then he can accept $x_{2}$ or choose a $\lambda^{2}$-proportional allocation $X^{2}$ over the partition $P^{c}$. By our tie-breaking rule, he accepts if and only if $u_{2}\left(x_{2}\right) \geq v_{2}\left(b_{2} \mid P^{c}\right)$. Anticipating this, if 2 is going to accept, then agent 3 accepts $x_{3}$ if and only if $u_{3}\left(x_{3}\right) \geq v_{3}\left(b_{3} \mid P^{c}\right)$. The result follows by induction.

Lemma 5 Assume that the common partition in any continuation game after a refusal is $P^{c}$. If the proposed allocation $X$ is refused, the final allocation is a $\lambda^{n}$-proportional $X^{n}$ chosen by player $n$ (who moves second).

Proof Suppose that $X$ is not accepted and let $i$ be the smallest index in $\{2, \ldots, n\}$ such that $u_{i}\left(x_{i}\right)<v_{i}\left(b_{i} \mid P^{c}\right)$. By Lemma $4, i$ refuses $X$ if the game reaches him. Anticipating this, agent $i+1$ (if any) has a choice between getting a proportional bundle of size $\lambda_{i+1}^{i}$ over the partition $P^{c}$ (if he accepts and lets the game reach $i$ ) or a proportional bundle of size $b_{i+1}$ over the same partition $P^{c}$ (if he refuses). But $\lambda_{i+1}^{i} \leq b_{i+1}$, so the first option can never lead to a higher utility for $i+1$. Therefore, by our tie-breaking rule, he prefers to stop the game. The result follows by induction.

The next two lemmata describe the optimal strategy for the divider, given a vector $\mathbf{b}=\left(b_{1}, \ldots, b_{n}\right)=\left(b_{1}, \mathbf{b}_{-1}\right)$ of bids from the first stage. Whenever there is a refusal, the proportionality coefficient for the divider is $\beta_{1}=\max \left(0,1-\sum_{s=2}^{n} b_{s}\right)$, regardless of the refuser $i$ 's identity. Since the common partition after a refusal is $P^{c}$, the divider's utility is $v_{1}\left(\beta_{1} \mid P^{c}\right)$. On the other hand, by Lemma 4 , the allocation $X$ is accepted if and only if $u_{i}\left(x_{i}\right) \geq v_{i}\left(b_{i} \mid P^{c}\right)$ for every $i \geq 2$. Let

$$
\mathcal{A}\left(\mathbf{b}_{-1}\right)=\left\{X \in \mathcal{A}: u_{i}\left(x_{i}\right) \geq v_{i}\left(b_{i} \mid P^{c}\right) \text { for each } i \geq 2\right\}
$$

be the set of allocations which are accepted.

Lemma 6 Assume that the common partition in any continuation game after a refusal is $P^{c}$. For any $\mathbf{b}_{-1}$ such that $\mathcal{A}\left(\mathbf{b}_{-1}\right) \neq \emptyset$ there exists an allocation which maximizes $u_{1}\left(x_{1}\right)$. A maximizing allocation $X^{*}\left(\mathbf{b}_{-1}\right)$ satisfies $u_{i}\left(x_{i}^{*}\right)=v_{i}\left(b_{i} \mid P^{c}\right)$ for each $i \geq 2$.

Proof Let $S\left(\mathbf{b}_{-1}\right)=[0,1] \times\left[v_{2}\left(b_{2} \mid P^{c}\right), 1\right] \times \cdots \times\left[v_{n}\left(b_{n} \mid P^{c}\right), 1\right]$ denote the cartesian product of $n$ intervals. This is a compact and convex subset of $\mathbb{R}^{n}$. Similarly, the partition range $R P(u)$, which spans the vector of utilities that the agents can achieve under a feasible allocation, is a nonempty, compact and convex subset of $\mathbb{R}^{n}$.

When $\mathcal{A}\left(\mathbf{b}_{-1}\right) \neq \emptyset$, there exists at least one allocation which maps to a vector of utilities in $S\left(\mathbf{b}_{-1}\right)$. Hence, the intersection of $R P(u)$ and $S\left(\mathbf{b}_{-1}\right)$ is not empty. As this 
intersection is also compact (and convex), there exists (at least) an allocation $X^{*}\left(\mathbf{b}_{-1}\right)$ which maximizes $u_{1}\left(x_{1}\right)$.

Now, suppose that at $X^{*}$ there is some $i \geq 2$ such that $u_{i}\left(x_{i}^{*}\right)>v_{i}\left(b_{i} \mid P^{c}\right)$. By the nonatomicity of $u_{i}$, we can always cut away a morsel from $x_{i}^{*}$ and reduce the utility of $i$ down to $v_{i}\left(b_{i} \mid P^{c}\right)$, transferring the morsel to agent 1's parcel. By mutual absolute continuity of preferences, this strictly increases the utility of agent 1 and therefore $X^{*}$ cannot be optimal. Therefore, at an optimal allocation $X^{*}$, the equality $u_{i}\left(x_{i}^{*}\right)=v_{i}\left(b_{i} \mid P^{c}\right)$ must hold for each $i \geq 2$.

Given $\mathbf{b}_{-1}$, the divider faces the choice of selecting an allocation which is accepted by everybody or another allocation which is eventually refused. In this second case, the common partition after a refusal is $P^{c}$ and then his utility is $v_{1}\left(\beta_{1} \mid P^{c}\right)$. Clearly, if $\mathcal{A}\left(\mathbf{b}_{-1}\right)=\varnothing$, there exists no acceptable allocation $X^{*}$ so the divider ends up with $v_{1}\left(\beta_{1} \mid P^{c}\right)$. Instead, if $\mathcal{A}\left(\mathbf{b}_{-1}\right) \neq \emptyset$, he proposes $X^{*}\left(\mathbf{b}_{-1}\right)$ if and only if $u_{1}\left(x_{1}^{*}\right) \geq$ $v_{1}\left(\beta_{1} \mid P^{c}\right)$. The next lemma summarizes this. Let $f\left(\mathbf{b}_{-1}\right)=\max \left\{u_{1}\left(x_{1}\right): X \in\right.$ $\left.\mathcal{A}\left(\mathbf{b}_{-1}\right)\right\}$, with the usual clause that $f\left(\mathbf{b}_{-1}\right)=-\infty$ if $\mathcal{A}\left(\mathbf{b}_{-1}\right)=\emptyset$. By Lemma 6 , this is well-defined and moreover, on $\mathcal{A}\left(\mathbf{b}_{-1}\right) \neq \emptyset$,

$$
f\left(\mathbf{b}_{-1}\right)=\max \left\{u_{1}\left(x_{1}\right): X \in \mathcal{A} \text { and } u_{i}\left(x_{i}\right)=v_{i}\left(b_{i} \mid P^{c}\right) \text { for each } i \geq 2\right\} .
$$

Lemma 7 Assume that the common partition in any continuation game after a refusal is $P^{c}$. For any $\mathbf{b}_{-1}$, agent 1 proposes an acceptable allocation $X^{*}\left(\mathbf{b}_{-1}\right)$ and the vector of final equilibrium payoffs is

$$
\left(f\left(\mathbf{b}_{-1}\right), v_{2}\left(b_{2} \mid P^{c}\right), \ldots, v_{n}\left(b_{n} \mid P^{c}\right)\right)
$$

if and only if $f\left(\mathbf{b}_{-1}\right) \geq v_{1}\left(\beta_{1} \mid P^{c}\right)$. Otherwise, agent 1 's proposal is refused by agent $n$ and the vector of final equilibrium payoffs is

$$
\left(v_{1}\left(\beta_{1} \mid P^{c}\right), v_{2}\left(\lambda_{2}^{n} \mid P^{c}\right), \ldots, v_{n}\left(\lambda_{n}^{n} \mid P^{c}\right)\right)
$$

with $\lambda_{i}^{n} \leq b_{i}$ for each $i \geq 2$.

The next lemma notes two useful properties for $f\left(\mathbf{b}_{-1}\right)$.

Lemma 8 Let $D$ be the interior set of $\left\{\mathbf{b}_{-1}: \mathcal{A}\left(\mathbf{b}_{-1}\right) \neq \emptyset\right\}$. The function $f\left(\mathbf{b}_{-1}\right)$ is (component-wise) strictly decreasing and continuous on $D$.

Proof By definition, $f\left(\mathbf{b}_{-1}\right) \geq 0$ on $D$. Recall that $v_{i}\left(b_{i} \mid P^{c}\right)$ is a strictly increasing and continuous function of $b_{i}$ for each $i$. By strict monotonicity, $b_{i}<b_{i}^{\prime}$ for some $i \geq 2$ implies $v_{i}\left(b_{i} \mid P^{c}\right)<v_{i}\left(b_{i}^{\prime} \mid P^{c}\right)$. If $\left(b_{2}, \ldots, b_{i} \ldots, b_{n}\right)$ and $\left(b_{2}, \ldots, b_{i}^{\prime}, \ldots, b_{n}\right)$ are in $D$, by (3) and mutual absolute continuity of preferences, $f\left(b_{2}, \ldots, b_{i} \ldots, b_{n}\right)>$ $f\left(b_{2}, \ldots, b_{i}^{\prime} \ldots, b_{n}\right)$.

To prove continuity, let $\mathbf{u}=\left(u_{1}, \ldots, u_{n}\right)$ denote a vector of utilities for each player and observe that

$$
f\left(\mathbf{b}_{-1}\right)=\max \left\{u_{1}: \mathbf{u} \in R P(u) \text { and } u_{i}=v_{i}\left(b_{i} \mid P^{c}\right) \text { for each } i \geq 2\right\} .
$$


For $\mathbf{b}_{-1}$ in $D$, let $\Gamma\left(\mathbf{b}_{-1}\right)=\left\{\mathbf{u} \in R P(u): u_{i}=v_{i}\left(b_{i} \mid P^{c}\right)\right.$ for each $\left.i \geq 2\right\}$ be the correspondence mapping $\mathbf{b}_{-1}$ into $R P(u)$. Since $R P(u)$ is nonempty, compact and convex, the continuity of each $v_{i}$ implies that $\Gamma\left(\mathbf{b}_{-1}\right)$ is compact-valued and continuous. Hence, by the Maximum theorem, the function $f\left(\mathbf{b}_{-1}\right)=\max \left\{u_{1}: \mathbf{u} \in \Gamma\left(\mathbf{b}_{-1}\right)\right\}$ is continuous.

The next lemma pins down equilibrium behavior in the first stage.

Lemma 9 Assume that the common partition in any continuation game after a refusal is $P^{c}$. The only possible equilibrium move in the first stage is that everyone makes the same bid

$$
b^{*}=\max \left\{\lambda: X \in \mathcal{A} \text { and } u_{i}\left(x_{i}\right) \geq v_{i}\left(\lambda \mid P^{c}\right) \text { for each } i\right\} .
$$

Proof First, we show that $b^{*}$ is well-defined. Let $\times_{i=1}^{n}\left[v_{i}\left(\lambda \mid P^{c}\right), 1\right]$ be the cartesian product of the $n$ intervals $\left[v_{i}\left(\lambda \mid P^{c}\right), 1\right]$, for $i=1, \ldots, n$. For any $\lambda$ in $[1 / n, 1]$, let $C(\lambda)=\times_{i=1}^{n}\left[v_{i}\left(\lambda \mid P^{c}\right), 1\right] \bigcap R P(u)$. By the proof of Theorem $2, C(1 / n) \neq \emptyset$. Moreover, $\lambda_{1}>\lambda_{2}$ implies $C\left(\lambda_{1}\right) \subset C\left(\lambda_{2}\right)$. Since $C(\lambda)$ is a decreasing collection of nested compact subsets, the finite intersection property implies that, for $\lambda^{*}=$ $\sup \{\lambda: C(\lambda) \neq \emptyset\}, C\left(\lambda^{*}\right) \neq \emptyset$. Clearly, $\lambda^{*}$ is equal to $b^{*}$.

Second, we show that if everybody bids $b^{*}$ then the divider proposes an acceptable allocation. By Lemma 7 , the divider does so if and only if $f\left(\mathbf{b}_{-1}\right) \geq v_{1}\left(\beta_{1} \mid P^{c}\right)$. By Theorem 2, $f\left(\mathbf{b}_{-1}\right) \geq v_{1}\left(1 / n \mid P^{c}\right)$. Moreover, $\beta_{1} \leq 1 / n$ and $v_{1}\left(\cdot \mid P^{c}\right)$ is an increasing function, so $f\left(\mathbf{b}_{-1}\right) \geq v_{1}\left(1 / n \mid P^{c}\right) \geq v_{1}\left(\beta_{1} \mid P^{c}\right)$.

Third, we show that an equilibrium move in the first stage cannot have $b_{i}<b_{1}$ for some $i$. Since $b_{1} \geq b_{i}$ for each $i$ by construction, this implies that the equilibrium bids must all be equal. Indeed, suppose $b_{i}<b_{1}$ for some $i$. There are two cases. If $\beta_{1}>0$, then $1-\beta_{1}=\sum_{i=2}^{n} b_{i}<1$. By choosing a bid $b_{i}^{\prime}$ such that $b_{i}<b_{i}^{\prime}<b_{1}$ and $b_{i}^{\prime}+\sum_{j \neq 1, i} b_{j}<1, i$ remains chooser and strictly increases his payoff to $v_{i}\left(b_{i}^{\prime} \mid P^{c}\right)$. If $\beta_{1}=0$, the inequality in the paragraph above strengthens to $f\left(\mathbf{b}_{-1}\right) \geq v_{1}\left(1 / n \mid P^{c}\right)>v_{1}\left(\beta_{1} \mid P^{c}\right)$ by the strict monotonicity of $v_{1}$. Then $\mathbf{b}_{-1}$ is in $D$ and by Lemma 8 agent $i$ can find a bid $b_{i}^{\prime}$ with $b_{i}<b_{i}^{\prime}<b_{1}$ and $f\left(b_{2}, \ldots, b_{i}^{\prime} \ldots, b_{n}\right)>v_{1}\left(\beta_{1} \mid P^{c}\right)$. The divider is still agent 1 who chooses an acceptable allocation, which strictly increases $i$ 's payoff to $v_{i}\left(b_{i}^{\prime} \mid P^{c}\right)$. In either case, $b_{i}$ is not a best reply.

The last paragraph implies that in equilibrium all bids are equal to a common value $b$. We now prove that $b=b^{*}$. Let $w_{i}(b)=\max \left\{u_{i}\left(x_{i}\right): X \in \mathcal{A}\right.$ and $u_{j}\left(x_{j}\right) \geq v_{j}\left(b \mid P^{c}\right)$ for $\left.j \neq i\right\}$. In other words, let $w_{i}(b)$ be the payoff to $i$ if he is the divider and everybody has made the same bid $b$. Clearly, $w_{i}(b)<v_{i}\left(b \mid P^{c}\right)$ if and only if $b>b^{*}$. Suppose that the common equilibrium bid is $b<b^{*}$. By the continuity of $w_{i}$, chooser $i$ can slightly raise his bid to $b^{\prime}$ and become the divider, which gets him a payoff $w_{i}\left(b^{\prime}\right)>v_{i}\left(b \mid P^{c}\right)$ so that $b$ is not a best reply. Now, suppose that the common equilibrium bid is $b>b^{*}$. Then the divider's payoff is $w_{1}(b)<v_{1}\left(b \mid P^{c}\right)$. Again, by continuity of $w_{1}$, he can slightly lower his bid to $b^{\prime}$ and become a chooser, which gets him a payoff $v_{1}\left(b^{\prime}\right)>w_{1}\left(b \mid P^{c}\right)$ so that $b$ is not a best reply. Hence, the only possible equilibrium must have everybody bidding $b^{*}$. Using Lemma 6 and the fact 
that $w_{i}(b)<v_{i}\left(b \mid P^{c}\right)$ if and only if $b>b^{*}$, it follows that when everybody bids $b^{*}$ each agent $i$ (regardless of his role as divider or chooser) receives a payoff $v_{i}\left(b^{*} \mid P^{c}\right)$ and thus he is indifferent between the two roles. Therefore, everybody bidding $b^{*}$ is the (only) equilibrium.

This completes our characterization of the equilibrium. In fact, we have shown a bit more. Since Lemmata 4-9 hold for any equilibrium where the common partition in any continuation game after a refusal is $P^{c}$, we know that the equilibrium payoff of player $i$ is $v_{i}\left(b^{*} \mid P^{c}\right)$ for this whole class of equilibria. Moreover, the proof shows also in equilibrium that the divider proposes an acceptable allocation that satisfies (3) and hence the payoffs are efficient. We record this in the following lemma.

Lemma 10 Assume that the common partition in any continuation game after a refusal is $P^{c}$. Then the only possible equilibrium outcome is efficient.

The next lemma exploits the efficiency of this equilibrium outcome to prove that there are no other equilibrium outcomes. The key observation is that each player alone can enforce the common partition $P^{c}$. Hence, each player $k$ holds a form of veto power on the common partition used after a refusal from another agent. A second observation is that Theorem 2 implies that each player can secure a utility level $\bar{u}=(1 / n)>0$; hence, in any equilibrium the divider cannot have zero utility.

Lemma 11 The equilibrium payoffs are unique, regardless of how the continuation game after a refusal is played.

Proof Suppose that there exists another putative equilibrium $E$ that is not payoff equivalent. Let $u_{i}^{*}=v_{i}\left(b^{*} \mid P^{c}\right)$ be the payoff obtained by player $i$ in any equilibrium where the common partition in any continuation game after a refusal is $P^{c}$. Since $E$ is not payoff equivalent to an efficient equilibrium, there exists some player $k$ who obtains a utility $u_{k}<u_{k}^{*}$. We show that this must lead to a contradiction. There are three distinct cases to examine.

Case 1 Suppose $b_{i} \geq b^{*}$ for all $i$. If player $k$ is getting utility $u_{k}<u_{k}^{*}$, there exists $\varepsilon>0$ such that $u_{k}<v_{k}\left(b^{*}-\varepsilon \mid P^{c}\right)<u_{k}^{*}$. Then player $k$ has a profitable deviation: bid $b^{*}-\varepsilon$ in stage 1 , reject any proposal in stage 2 and, in case of refusal, announce the natural partition $P^{c}$. This makes player $k$ the first chooser and gives him a utility $v_{k}\left(b^{*}-\varepsilon \mid P^{c}\right)>u_{k}$.

Case 2 Suppose $b_{i} \leq b^{*}$ for all $i$. Again, player $k$ has a profitable deviation: bid $b_{k}>b^{*}$ in stage 1 , propose an appropriate allocation that give each agent $i \neq k$ a utility $u_{i}^{*}$ and announce $P^{c}$ in case of refusal. Since this higher bid gives $k$ the right to be divider and indifferent choosers prefer to accept, any allocation that satisfies (3) gives him a utility $u_{k}^{*}>u_{k}$.

Case 3 Suppose $b_{1}>b^{*}>b_{n}$. Similarly to the third part of the proof of Lemma 9, we show that this cannot be an equilibrium. Given the tie-breaking order $b_{1} \geq b-2 \geq$ $\cdots \geq b_{n}$, let $m$ be the first agent who has bid $b_{n}$. (If only one agent has bid $b_{n}$, then clearly $m=n$.) 
First, assume that in equilibrium the allocation proposed by the divider is accepted. Clearly, chooser $m$ must get at least $v_{m}\left(b_{m} \mid P^{c}\right)$; otherwise, he could profitably deviate to refusing and announcing $P^{c}$. On the other hand, by mutual absolute continuity of preferences, player $m$ gets no more than $v_{m}\left(b_{m} \mid P^{c}\right)$; otherwise, the divider could increase his own payoff by appropriating a morsel from $m$ 's parcel, reducing his utility exactly to $v_{m}\left(b_{m} \mid P^{c}\right)$ and announcing $P^{c}$ in case of refusal. By Theorem 2 , the divider has a strictly positive utility in equilibrium and therefore is left with a parcel from which $m$ can claim an additional morsel. In fact, there is a sufficiently small $\varepsilon>0$ such that player $m$ can profitably deviate to a higher bid $b_{m}+\varepsilon<b_{m-1}$, announcing $P^{c}$ in case of refusal. If $m=n$, the player remains the first chooser and is guaranteed a utility $v_{m}\left(b_{m}+\varepsilon \mid P^{c}\right)>v_{m}\left(b_{m} \mid P^{c}\right)$. If $m<n$ and $\varepsilon$ is sufficiently small, there still exists an efficient allocation that is accepted by all choosers so the divider's best reply is to make a proposal that is not rejected.

Second, assume that in equilibrium the allocation proposed by the divider 1 is refused. Again, by Theorem 2, the divider has a strictly positive utility in equilibrium and therefore is left with a parcel from which $m$ can claim an additional morsel by deviating to a higher bid $b_{m}+\varepsilon<b_{m-1}$ and announcing $P^{c}$ in case of refusal.

The proof of the main result follows.

Proof of Theorem 3. Lemma 11 shows that equilibrium payoffs are unique. Lemmas 10 and 9 prove that the equilibrium outcome is efficient and egalitarian equivalent, respectively. The proof of Lemma 9 also shows that each agent $i$ is indifferent between the parcel received and the $\lambda^{*} 1$-proportional allocation.

\section{References}

Akin, E.: Vilfredo Pareto cuts the cake. J Math Econ 24, 23-44 (1995)

Barbanel, J.: The Geometry of Efficient Fair Division. Cambridge: Cambridge University Press (2005)

Barbanel, J., Zwicker, W.S.: Two applications of a theorem of Dvoretzky, Wald, and Wolfowitz. Theory Decis 43, 203-207 (1997)

Berliant, M., Thomson, W., Dunz, K.: On the fair division of a heterogeneous commodity. J Math Econ 21, 201-206 (1992)

Berliant, M., Dunz, K.: A foundation of location theory: Existence of equilibrium, the welfare theorems, and core. J Math Econ 40, 593-618 (2004)

Chambers, C.P.: Allocation rules for land division. J Econ Theory 121, 236-258 (2005)

Crawford, V.P.: A procedure for generating Pareto-efficient egalitarian-equivalent allocations. Econometrica 47, 49-60 (1979)

Demange, G.: Implementing efficient egalitarian equivalent allocations. Econometrica 52, 1167-1177 (1984)

Dvoretzky, A., Wald, A., Wolfowitz, A.: Relations among certain ranges of vector measures. Pac J Math 1, 59-74 (1951)

Dubins, L.E., Spanier, E.H.: How to cut a cake fairly. Am Math Monthly 68, 1-17 (1961)

Foley, D.: Resource allocation and the public sector. Yale Econ Essays 7, 45-98 (1967)

Gamow, G., Stern, M.: Puzzle-Math. New York: The Viking Press (1958)

Maccheroni, F., Marinacci, M.: How to cut a pizza fairly: Fair division with decreasing marginal evaluations. Soc Choice Welf 2, 457-65 (2003)

Miceli, T.J., Sirmans, C.F.: Partition of real estate; or breaking up is (not) hard to do. J Leg Stud 29, 783796 (2000)

Pazner, E.A., Schmeidler, D.: Egalitarian equivalent allocations: A new concept of economic equity. Q J Econ 92, 671-687 (1978) 
Sprumont, Y.: What is a commodity? Two axiomatic answers. Econ Theory 23, 429-437 (2004) Sprumont, Y., Zhou, L.: Pazner-Schmeidler rules in large societies. J Math Econ 31, 321-339 (1999)

Steinhaus, H.: The problem of fair division. Econometrica 16, 101-104 (1948)

Stromquist, W.: How to cut a cake fairly. Am Math Monthly 87, 640-644 (1980)

Thomson, W.: Divide-and-permute. Games Econ Beh 52, 186-200 (2005)

Weller, D.: Fair division of a measurable space. J Math Econ 14, 5-17 (1985) 